geld. Offenbar beruft sich der Kl. damit auf das Regulierungsverhalten des Bekl. bzw. des hinter ihm stehenden Haftpflichtversicherers. Zwar kann ein zögerliches Regulierungsverhalten bedeutsam für die Höhe des zuzuerkennenden Schmerzensgeldes sein. Ein Ausgleichszuschlag kommt aber nur dann in Betracht, wenn ein schützenswertes Interesse an der prozessualen Haltung der beklagten Partei nicht besteht, weil die Rechtslage eindeutig ist, der Schädiger also grundlos die Entschädigungszahlung verzögert und davon auszugehen ist, dass durch das $\mathrm{Re}-$ gulierungsverhalten des Leid des geschädigten Patienten in der Gesamtschau erhöht und deshalb ein zusätzlicher Ausgleich zu gewähren ist vgl. etwa OLG Naumburg, VersR 2002, 1295; Wenzel/Hensen, Der Arzthaftungsprozess, Rdnr. 2509). $\mathrm{Zu}$ diesen Voraussetzungen verhält sich das Vorbringen des Kl. nicht.

Ebenso wenig kommt eine Erhöhung des Schmerzensgeldes aufgrund des bestehenden Haftpflichtversicherungsschutzes in Betracht. Das Bestehen einer Haftpflichtversicherung führt nur dazu, dass es für die Frage der Leistungsfähigkeit, die bei der Schmerzensgeldzumessung zugunsten des Schädigers berücksichtigt werden kann, nicht mehr auf die Vermögensverhältnisse des Schädigers ankommt, erhöht aber nicht die Haftung des Schädigers (vgl. nur Koch, in: Bruck/Möller, VVG, 9. Aufl. 2013, Vorbemerkung zu $\$ S$ 100-112,Rdnr. 81). [...]

DOI: 10.1007/s00350-017-4605-1

\section{Anmerkung zu OLG Koblenz, Beschl. v. 6.1.2016 - 5 U 1148/15}

\section{Lothar Jaeger}

Die Entscheidung zur Höhe des Schmerzensgeldes ist auf der Grundlage der in vergleichbaren Fällen ausgeurteilten Schmerzensgeldbeträge nicht unbedingt zu beanstanden. Auch erhebliche Dauerschäden an Unterarm und Hand werden von der Rechtsprechung nicht immer mit einem angemessenen Schmerzensgeld ausgeglichen.

Dieser Umstand ist allerdings dem OLG Koblenz nicht alleine vorzuwerfen, er beruht vielmehr meist darauf, dass Anwälte und Richter, obwohl sie dazu in der Lage sein sollten, nicht genügend Phantasie entwickeln, um sich vorzustellen, welche Auswirkungen ein Dauerschaden an der rechten Hand für einen Winzer im Vergleich zu den am Verfahren beteiligten Rechtsanwälten und Richtern hat. Das zeigen auch die in den Entscheidungssammlungen mitgeteilten Entscheidungen. In fast allen Urteilen fehlt eine eingehende Schilderung des Alltagslebens der Betroffenen. Das liegt daran, dass die Geschädigten nicht wissen, warum ihr Rechtsanwalt und die Richter nicht verstehen, was es für einen „Arbeiter“ bedeutet, wenn er „durch die Einschränkung der Funktion der rechten Hand mit erheblichen Einschränkungen konfrontiert" wird. Volljuristen haben diese Probleme allenfalls bei einer Hobbytätigkeit. Schon die Wahl der Ausdrucksweise zeigt, dass die Richter nicht verstanden haben können, was der Winzer über seinen Anwalt, der ihn auch nicht verstanden hatte, mitteilen wollte. Was bedeutet die Feststellung im Urteil, dass der Kläger durch die „Reduzierung der Weinbergsflächen“ geringere Einnahmen habe? Warum genau hat der Kläger die Flächen reduzieren müssen? Das wird auch nicht deutlich durch den weiteren Satz im Urteil, ,verletzungsbedingt erfolgte die Umstellung seiner Weinproduktion auf die ,... Vermarktung von Fasswein".

VRiOLG a. D. Lothar Jaeger,

Mechtildisstraße 3, 50678 Köln, Deutschland
Juristen sehen in einem solchen Sachvortrag in erster Linie die wirtschaftlichen Folgen einer Behinderung, nicht aber den Körper- und Gesundheitsschaden selbst, der durch das Schmerzensgeld ausgeglichen werden soll. Sie sehen nicht die psychische Beeinträchtigung - auch bedingt durch den Ansehensverlust des klagenden Winzers bei dessen Kollegen. Es sei zwar richtig, dass die mit der unfallbedingten Behinderung bei der Berufsausübung verbundenen seelischen Beeinträchtigungen bestehen könnten. Die - seelischen Beeinträchtigungen - setzt der Senat in Parenthese und gerade weil der Senat dem Vortrag des Klägers, ,er leide an einem geringeren Ansehen in seiner Berufsgruppe“ in dieser Pauschalität keinen schmerzensgelderhöhenden Charakter zu entnehmen vermag, zeigt, dass er das seelische Leid des Klägers nicht erfasst und verstanden hat, und dass er den Kläger zu einer mündlichen Verhandlung hätte laden sollen.

Aus alledem folgt nicht, dass der Anwalt des Klägers und die Richter gewusst haben, welche Arbeiten der Kläger verletzungsbedingt hat umstellen müssen, weil er sie nicht mehr ausüben konnte. Gefragt haben sie den Kläger allerdings nicht. Stattdessen verlangen die Richter des OLG Koblenz vom Kläger die zur Zumessung (gemeint wohl: Bemessung) des Schmerzensgeldes aus seiner Sicht maßgebenden (schmerzensgeldbegründenden und -erhöhenden) Umstände vorzutragen. Im Urteil teilen sie dann mit, dass der Kläger zutreffende Bemessungsfaktoren angeführt habe, die für die Höhe des Schmerzensgeldes relevant seien. Das ist keine Subsumtion. Worin bestehen denn die „ohne weiteres nachvollziehbaren Einschränkungen bei der Entfaltung der erforderlichen Tätigkeiten als Winzer"? Was bedeutet die Umstellung der Produktion auf Fasswein, welche Fähigkeiten fehlen dem Kläger, um weiterhin Flaschenwein zu produzieren? Alles bleibt dunkel, alles wird mit Floskeln abgetan.

Daraus folgt: $\mathrm{Zu}$ beanstanden ist nicht nur die unzureichende Substantiierung durch den Kläger, sondern die unzureichende Sachverhaltsaufklärung durch den Senat und seine scheinbar sorgfältige Anwendung der ,pauschalen Schilderungen des Klägers" auf den Fall.

Hätte der Senat verhandelt und das persönliche Erscheinen des Klägers angeordnet, hätte er binnen weniger Minuten erfahren, was den Kläger bedrückt und aus welchen körperlichen Defiziten er den höheren Schmerzensgeldanspruch herleitet. Stattdessen wird dem Kläger vorgeworfen, er habe sich - ebenso wie das LG -sic.- auf eine pauschale Erwähnung der maßgebenden Bemessungsfaktoren beschränkt und diese Pauschalierung betreffe auch die Berufungsbegründung des Klägers, in der etwa auf Schmerzen und Einschränkungen abgestellt werde, ohne diese näher auszuführen. Wie geht denn das, obwohl das LG nach der Feststellung des Senats die maßgeblichen Bemessungsfaktoren berücksichtigt hat, obwohl der Kläger sogar in der Berufungsbegründung auf Schmerzen und Einschränkungen abgestellt hat, ohne diese näher auszuführen? Haben die Richter des Senats wirklich die Sachkunde, alles hinzuzudenken, was das LG pauschaliert und was sogar der Kläger nicht vorgetragen, sondern nur pauschaliert hat? Nein, der Senat hat ebenso pauschal geurteilt wie der Kläger nach Ansicht des Senats unzureichend pauschal vorgetragen hat.

Dennoch bedeutet dies nicht zwingend, dass das Schmerzensgeld der Höhe nach unzutreffend ist. Der Sachverhalt ist nicht hinreichend aufgeklärt, was natürlich auch dem Anwalt des Kl. vorzuwerfen ist.

Solange Fragen offen sind, sollte ein Senat mündlich verhandeln, die Parteien und deren Anwälte anhören und den realen Sachverhalt und nicht den prozessual vermeintlich ausreichenden entscheiden. Ein wenig mehr Mühe kann zur Befriedung beitragen und mancher Partei zum Recht verhelfen. 